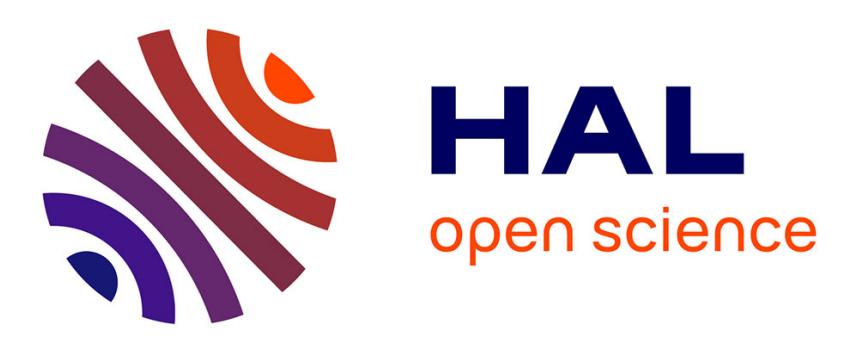

\title{
Mechanical work performed by individual limbs of transfemoral amputees during step-to-step transitions: Effect of walking velocity
}

Xavier Bonnet, Coralie Villa, Pascale Fode, Francois Lavaste, Helene Pillet

\section{- To cite this version:}

Xavier Bonnet, Coralie Villa, Pascale Fode, Francois Lavaste, Helene Pillet. Mechanical work performed by individual limbs of transfemoral amputees during step-to-step transitions: Effect of walking velocity. Journal of Engineering in Medicine, 2014, 228 (1), pp.60-66. 10.1177/0954411913514036 . hal-01083626

\section{HAL Id: hal-01083626 \\ https://hal.science/hal-01083626}

Submitted on 2 Apr 2015

HAL is a multi-disciplinary open access archive for the deposit and dissemination of scientific research documents, whether they are published or not. The documents may come from teaching and research institutions in France or abroad, or from public or private research centers.
L'archive ouverte pluridisciplinaire $\mathbf{H A L}$, est destinée au dépôt et à la diffusion de documents scientifiques de niveau recherche, publiés ou non, émanant des établissements d'enseignement et de recherche français ou étrangers, des laboratoires publics ou privés. 


\title{
Mechanical work performed by individual limbs of transfemoral amputees during step-to-step transitions: Effect of walking velocity
}

\author{
Xavier Bonnet', Coralie Villa', Pascale Fodé2, Francois Lavaste ${ }^{1,2}$ \\ and Hélène Pillet ${ }^{\prime}$
}

\begin{abstract}
The greater metabolic demand during the gait of people with a transfemoral amputation limits their autonomy and walking velocity. Major modifications of the kinematic and kinetic patterns of transfemoral amputee gait quantified using gait analysis may explain their greater energy cost. Donelan et al. proposed a method called the individual limb method to explore the relationships between the gait biomechanics and metabolic cost. In the present study, we applied this method to quantify mechanical work performed by the affected and intact limbs of transfemoral amputees. We compared a cohort of six active unilateral transfemoral amputees to a control group of six asymptomatic subjects. Compared to the control group, we found that there was significantly less mechanical work produced by the affected leg and significantly more work performed by the unaffected leg during the step-to-step transition. We also found that this mechanical work increased with walking velocity; the increase was less pronounced for the affected leg and substantial for the unaffected leg. Finally, we observed that the lesser work produced by the affected leg was linked to the increase in the hip flexion moment during the late stance phase, which is necessary for initiating knee flexion in the affected leg. It is possible to quantify the mechanical work performed during gait by people with a transfemoral amputation, using the individual limb method and conventional gait laboratory equipment. The method provides information that is useful for prosthetic fitting and rehabilitation.
\end{abstract}

\section{Keywords}

Locomotion, prosthetics, biomechanics, hip moment, mechanical power

\section{Introduction}

People with transfemoral amputation expend more metabolic energy while walking than non-amputees. It is also known that the more proximal the amputation, the greater the energy consumption. ${ }^{1}$ In addition, Schmalz et al. have shown that the metabolic rate increases at faster walking speed and is greater in people with a leg amputation., The quantification of metabolic energy cost, as an evaluation of the overall efficiency of gait, has been widely used to compare prosthetic elements. ${ }^{3-6}$ However, the reference method of quantification, oxygen consumption, implies the measurement of exhaled gas and cannot always be easily integrated into conventional gait analysis.

Among possible explanations for the greater energy consumption of amputees, different coping mechanisms, impacting both kinematic and kinetic parameters of gait, have been described in the literature. The stance flexion of the prosthetic knee dramatically decreases and is often totally absent. Prosthetic feet do not allow a powered plantar flexion at the end of the stance phase, ${ }^{7}$ producing about $20 \%$ less push off work at the prosthetic ankle compared to nonamputees. ${ }^{8}$ Kinetic parameters of the contralateral joints are also modified, as a consequence of the lack

'Laboratoire de BioMécanique, Arts et Metiers ParisTech, Paris, France ${ }^{2} \mathrm{CERAH}$, Institution Nationale des Invalides, Woippy, France

\section{Corresponding author:}

Hélène Pillet, Laboratoire de BioMécanique, Arts et Metiers ParisTech, I5I bd de l'hopital, 750I3 Paris, France.

Email: helene.pillet@ensam.eu 
of prosthetic push off and to control the prosthetic knee. ${ }^{8-10}$ Modifications are especially observed during the double support phase of gait. Nolan and Lees ${ }^{11}$ showed an overuse of the patient's intact limb compared to normal ambulation. In particular, hip moments were greater for people with transfemoral amputation than for asymptomatic subjects. These moments involve increased hip extensor activity in early stance and increased hip flexor 'pull-off' activity by the affected limb during push off. ${ }^{8}$ Schmalz et al. ${ }^{2}$ also showed the influence of prosthetic alignment variations on the hip flexion moment necessary to initiate knee bending in late stance phase and hypothesized that the muscular effort needed was a cause for the increase in oxygen consumption. The calculation of mechanical work from gait analysis data may help us to understand the causes of the variations of metabolic energy expenditure.

It was hypothesized that the kinematic compensations during transfemoral amputee gait modify the centre of gravity path and can explain the metabolic inefficiency of above knee amputee gait. However, although the metabolic cost was $27 \%$ higher in amputees compared with normal subjects in the study by Gitter et al., ${ }^{12}$ no significant differences were noted in the mechanical work done on the trunk centre of mass. Moreover, Detrembleur et al. ${ }^{13}$ showed that even if the vertical displacement of the body centre of mass was much higher for transfemoral amputees due to the lack of knee damping, the energy cost was not directly affected thanks to the better efficiency of the pendulum-like mechanism during the single stance phase of gait. It may be that the major part of the energy loss occurs during the step-to-step transition, and thus, the calculation of external total energy of the centre of mass may not be sufficient to explain the variations in metabolic energy.

On the other hand, several inverted pendulum models of walking have been developed to investigate the metabolic cost of human walking from the mechanical point of view. ${ }^{14-16}$ Although the lower limb nearly behaves as a pendulum during single support phase, the need to redirect the centre of mass during double support requires muscular work. Thus, Kuo et al. ${ }^{16}$ showed that work was needed between steps rather than within each step.

In particular, Donelan et al. ${ }^{17}$ has shown that the mechanical work needed for the step-to-step transitions is a major determinant of the metabolic cost of walking of asymptomatic subjects. Regarding amputee gait, the step-to-step transition was evaluated by Houdijk et al. ${ }^{18}$ for transtibial amputees. They concluded that the increase of mechanical work needed for the transition from the affected limb to the intact limb contributed to the increased metabolic cost.

Until now, no study has investigated this transition for transfemoral amputees. However, it can be hypothesized that this transition is all the more difficult for transfemoral amputees due to the behaviour of their prosthetic knee and foot. This is particularly true during the transition between the affected limb and the intact limb. People with a transfemoral amputation must transfer their centre of mass on the intact limb and to initiate prosthetic knee flexion, in order to release the prosthetic knee joint. This hypothesis may be consistent with the observation of Schmalz et al. ${ }^{2}$ and Radcliffe et al. ${ }^{10}$ concerning the increase of hip flexion moment during the double stance of gait, considered as an active strategy to induce an external flexion moment at the prosthetic knee. In this framework, the aim of this study was to evaluate mechanical work performed during step-to-step transition for transfemoral amputees compared to able-bodied people at different walking velocities.

\section{Material and method}

\section{Subjects}

Six active male transfemoral amputees (age: $43 \pm 13$ years; height: $1.80 \pm 0.07 \mathrm{~m}$; mass: $79 \pm 6 \mathrm{~kg}$ ) and six control subjects (three males and three females: age 29 \pm 5 years; height: $1.77 \pm 0.13 \mathrm{~m}$; mass $72 \pm 13 \mathrm{~kg}$ ) participated in the study. All amputees used their own prostheses. All the prosthetic knees had a regulation of the swing phase, which was controlled by microprocessor for five of them. All patients were amputated for traumatic reasons and were active walkers. The protocol was approved by the local ethics committee and all subjects gave their consent.

\section{Protocol}

All experiments were performed on level ground. Each subject first adopted their self-selected walking speed (SSWS). This speed was identified by asking the subject to walk along the $9 \mathrm{~m}$ walkway at a comfortable velocity. Then, the subject was asked to walk more slowly on the same walkway, which allows determining his slow walking speed (SWS), and finally, he was asked to walk faster and his fast walking speed (FWS) was obtained. So, each subject performed the experiment at three different speeds. For each speed, at least five successful trials were done by the subjects. The walkway was equipped with two separate force plates (AMTI) in the middle of the path. A trial was considered successful when each lower limb hits the floor in one of the two plates. So, for each trial, three components of the ground reaction force and three components of the ground reaction moment at the centre of the plate were collected under each lower limb at a sampling rate of $120 \mathrm{~Hz}$. During these trials, segmental and articular kinematics were measured with an optoelectronic system (Vicon V8i 8 cameras) at a sampling rate of 120 Hz. Markers were positioned on specific anatomical landmarks in accordance with the protocol described by Pillet et al. ${ }^{19}$ 
In addition, a static acquisition was performed prior to the experiment in order to create a personalized geometric model of the body. The method used to obtain the geometric model was presented in details by Pillet et al. ${ }^{19}$

\section{Data analysis}

The average walking velocity was quantified by calculating the average velocity of a marker placed on the first thoracic vertebra. The position of the centre of mass of the body was obtained from the subject-specific geometric model. The volume of each body segment was computed and used to find the mass and the centre of mass of this segment. For sound segments, Dempster's ${ }^{20}$ densities were used. Prosthetic components were weighed. The density of each prosthetic segment was adjusted to match the segment mass and was used for the calculation of its centre of mass.

The three components of the velocity of the centre of mass of the body were calculated as the first derivative of its three-dimensional (3D) coordinates using a fourth-order finite difference. Prior to the derivation, the data were filtered with a zero-phase forward and reverse butterworth fourth-order filter at each step. The cut-off frequency was $5 \mathrm{~Hz}$.

Mechanical power was computed using the method put forward by Donelan et al. ${ }^{21}$ The mechanical powers produced by each lower limb were computed as the dot product of the ground reaction force applied on each limb and the body's centre of mass velocity (see Appendix 1 for more details). The mechanical work was then assessed using the time integral of the mechanical power profiles. In particular, the double support phases of gait were analysed. For each cycle of gait, two double support phases were defined: the mechanical work calculated over the first and the second double supports were called $\mathrm{WDS}_{\text {lead }}$ and $\mathrm{WDS}_{\text {trail, }}$ respectively, according to the notations proposed by Houdijk et al. ${ }^{18}$ They were divided by the body mass of each subject and expressed in Joule per kilogram. So, for people with amputations due to the asymmetry of gait, it was possible to calculate two values of each parameter depending on the leading lower limb of the cycle (prosthetic or contralateral).

To understand the link with lower limb kinetic adaptations, the peak hip moment during double support of the late stance phase was selected. Using inverse dynamic methods, hip moment was also computed from force plate and segmental kinematic data. From this curve, the maximum during the double support phase (corresponding to the peak of hip flexion moment) was calculated.

\section{Statistics}

Statistical analyses were performed using MATLAB. Descriptive statistics for each parameter were first performed. The influences of the speed and the limb

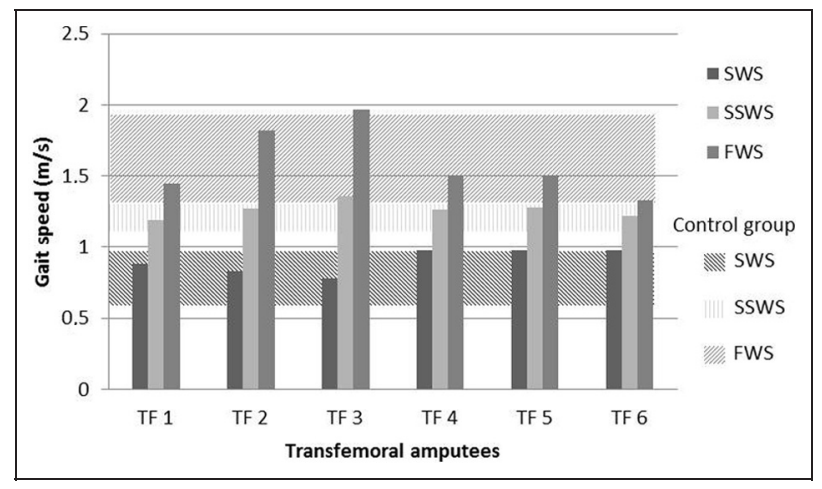

Figure I. Average walking speeds of each transfemoral subject compared to the range of speeds observed for the control group for the three walking conditions.

SWS: slow walking speed; SSWS: self-selected walking speed; FWS: fast walking speed.

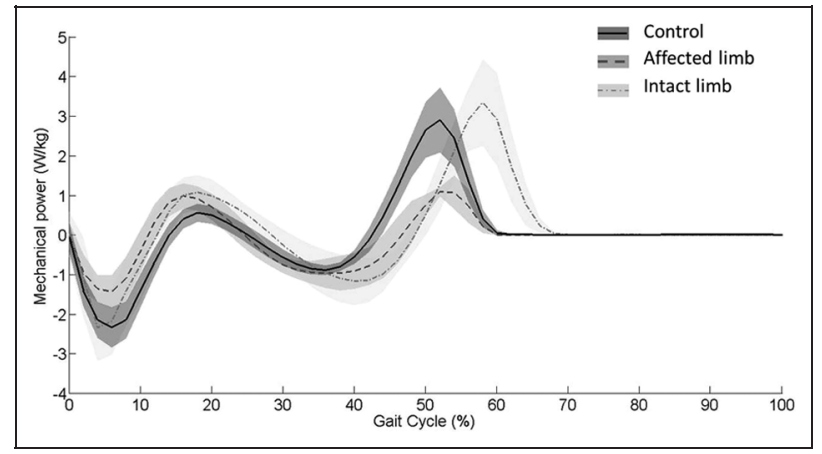

Figure 2. Mechanical power produced by affected limb (dashed line) and intact limb (dash-dotted line) of transfemoral amputees compared to asymptomatic subjects (solid line) (W/kg) according to the percentage of gait cycle in the self-selected walking speed condition.

(prosthetic, contralateral and control) were tested using a non-parametric test of Friedman for independent observations. Correlation between mechanical work and walking velocity was analysed using Pearson's correlation coefficient for each group (prosthetic leg, intact leg and control group). Statistical significance was set at $p<0.05$.

\section{Results \\ Walking velocity}

For the three walking conditions, Figure 1 shows that walking velocities achieved by transfemoral amputees were slower on average but in the range of speeds collected for the control group.

\section{Mechanical work}

Average mechanical power patterns generated by the affected limb, the contralateral limb and the limbs of asymptomatic subjects are depicted in Figure 2 for the 
Table I. Mechanical works $(\mathrm{J} / \mathrm{kg})$ at three different walking velocities.

\begin{tabular}{|c|c|c|c|c|}
\hline $\begin{array}{l}\text { Walking velocity } \\
\text { conditions }\end{array}$ & Parameters $(\mathrm{J} / \mathrm{kg})$ & $\begin{array}{l}\text { Amputee patients: } \\
\text { intact limb cycle }\end{array}$ & $\begin{array}{l}\text { Amputee patients: } \\
\text { prosthetic limb cycle }\end{array}$ & Control subjects \\
\hline \multirow[t]{2}{*}{ SWS } & $W^{\prime} S_{\text {lead }}$ & $-0.12(0.02)$ & NA & $-0.13(0.03)$ \\
\hline & $W_{\text {trail }}$ & $0.22(0.03)$ & NA & $0.25(0.05)$ \\
\hline \multirow[t]{2}{*}{ SSWS } & $W S_{\text {lead }}$ & $-0.19(0.09)$ & $-0.12(0.05)$ & $-0.18(0.04)$ \\
\hline & $W_{\text {trail }}$ & $0.34(0.10)$ & $0.09(0.03)$ & $0.28(0.06)$ \\
\hline \multirow[t]{2}{*}{ FWS } & $W^{\prime} S_{\text {lead }}$ & $-0.30(0.14)$ & $-0.23(0.14)$ & $-0.37(0.11)$ \\
\hline & $W_{\text {trail }}$ & $0.48(0.23)$ & $0.13(0.05)$ & $0.39(0.11)$ \\
\hline
\end{tabular}

SWS: slow walking speed; SSWS: self-selected walking speed; FWS: fast walking speed; NA: not available data; WDS lead $_{\text {and }}$ WDS trail: $_{\text {given for each }}$ limb cycle for amputee patients.

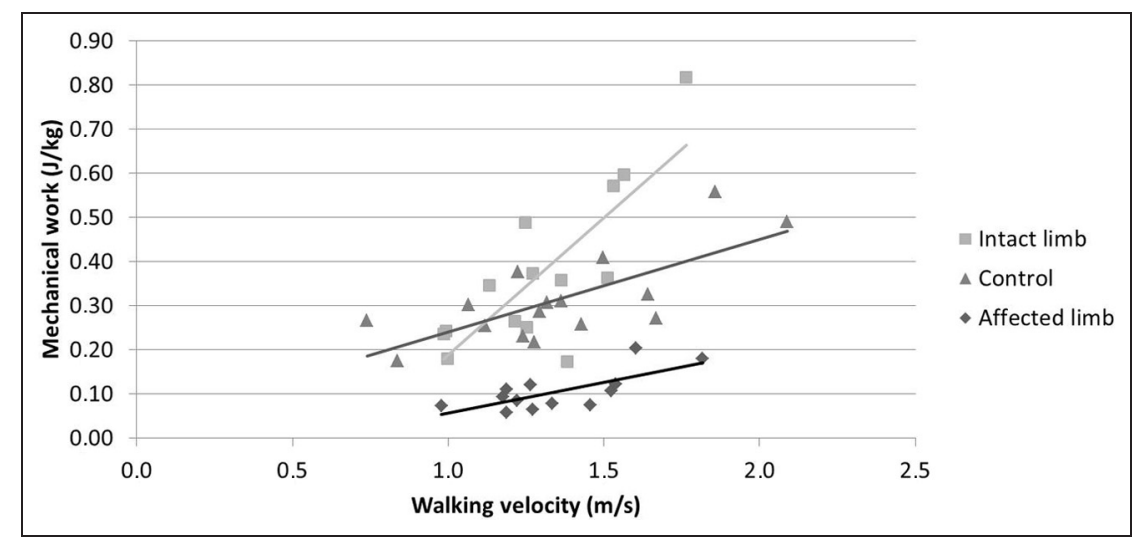

Figure 3. Mechanical work of trailing leg during the second double support produced by the affected limb (black diamond) and the intact limb (grey square) of transfemoral amputees compared to the one produced by the right limb of asymptomatic subjects (dark grey triangle) according to walking velocity.

SSWS. The shaded bands represent the interindividual variability assessed by \pm 1 standard deviation from the mean.

Mechanical work calculated over the first and the second double supports for each limb for each speed condition is provided in Table $1 . \mathrm{WDS}_{\text {lead }}$ and $\mathrm{WDS}_{\text {trail }}$ were available for all speeds for the intact limb but values at SWS could not be calculated for the affected limbs. Indeed, because of the slow walking velocity, the step lengths of the patients were too small to allow them to strike both force plates during these trials.

Friedman tests showed a significant influence of the speed at $p=0.02$ and a significant influence of the limb at $p=0.0001$ on the mechanical work of the trailing leg during double support time. The mechanical work provided by the affected limb during double support phases was always less than the one produced by the control group. On the contrary, the mechanical work produced by the intact limb at the end of the stance phase was much greater than that of the control group.

$\mathrm{WDS}_{\text {trail }}$ produced by the control group during the second double support phase increased with walking speed. This increase was lower for the affected limb and higher for the contralateral limb (Figure 3). The correlation between $\mathrm{WDS}_{\text {trail }}$ and walking speed was shown to be significant for the affected $\operatorname{limb}(\mathrm{r}=0.72, p=$ $0.008)$ and the contralateral limb $(\mathrm{r}=0.75, p=0.007)$ of the amputee and for the control group $(\mathrm{r}=0.72$, $p=0.008)$.

\section{Hip moment}

Mean and standard deviation of maximum hip flexion moments computed for able-bodied subjects and transfemoral amputees are reported in Table 2. For all speeds, the peak hip moment at the prosthetic side in the population of amputees was larger by between 0.10 and $0.20 \mathrm{~N} \mathrm{~m} / \mathrm{kg}$ than the one computed at the sound side. It was also larger than the one computed for the control group. Thus, the maximum hip moment was greater in transfemoral amputees on their affected limb.

\section{Discussion}

In this study, the aim was to investigate the mechanical work required by the individual limbs during step-tostep transition in a population of transfemoral amputees according to the walking velocity. A control group was used as a reference. The results of mechanical work during the step-to-step transition of the asymptomatic subjects $\left(\mathrm{WDS}_{\text {lead }}=-0.28(0.05) \mathrm{J} / \mathrm{kg}\right.$ and $\mathrm{WDS}_{\text {trail }}=$ $0.18(0.05) \mathrm{J} / \mathrm{kg}$ ) were consistent with the results of Houdijk et al. ${ }^{18}$ for transtibial amputees and for similar 
Table 2. Mean and standard deviation of the maximal hip extension moments $(\mathrm{N} \mathrm{m} / \mathrm{kg}$ ) for the three walking velocity conditions for both limbs of transfemoral amputees and control subjects.

\begin{tabular}{llll}
\hline $\begin{array}{l}\text { Maximum of external hip } \\
\text { extension moment }(\mathrm{N} \mathrm{m} / \mathrm{kg})\end{array}$ & Amputee: intact limb & Amputee: prosthetic limb & Control subject \\
\hline SWS & $0.51(0.10)$ & $0.72(0.17)$ & $0.57(0.17)$ \\
SSWS & $0.74(0.31)$ & $0.86(0.22)$ & $0.80(0.13)$ \\
FWS & $0.86(0.29)$ & $1.06(0.34)$ & $1.02(0.14)$ \\
\hline
\end{tabular}

SWS: slow walking speed; SSWS: self-selected walking speed; FWS: fast walking speed.

walking velocities. In accordance with the literature, the walking velocities chosen by people with amputation were slower on average than those of asymptomatic subjects. However, the subjects were active enough to be able to walk with speeds in the range observed in the control group.

Our results for transfemoral amputees were fundamentally similar in nature to those for transtibial amputees studied by Houdijk et al. ${ }^{18}$ but the effects were quantitatively much greater in the transfemoral amputees. The affected limb was able to produce limited mechanical work in late stance $\left(\mathrm{WDS}_{\text {trail }}=0.09\right.$ (0.03) $\mathrm{J} / \mathrm{kg}$ vs $0.16(0.04) \mathrm{J} / \mathrm{kg}$ for transtibial amputee $\left.^{18}\right)$. The mechanical work produced by the intact limb in late stance was much greater for transfemoral amputees $\left(\mathrm{WDS}_{\text {trail }}=0.34(0.10) \mathrm{J} / \mathrm{kg}\right)$ than for transtibial amputees $\left(\mathrm{WDS}_{\text {trail }}=0.27(0.04) \mathrm{W} / \mathrm{kg}^{18}\right)$. It confirmed the finding of Tesio et al. ${ }^{22}$ who observed a greater asymmetry of mechanical energy changes of the centre of mass for above knee amputees than for below knee amputees. The results showed that mechanical work produced by the prosthetic leg during the push off phase, when the intact limb was leading, was dramatically less compared to the control group. In parallel, the mechanical work of the intact leading limb increased. These results are consistent with previous study, which showed a decrease of the prosthetic ankle power generated during the propulsion and a greater concentric hip extensor work done in early stance for the contralateral limb. ${ }^{8}$ At the same time, the mechanical work produced during the push off of the intact limb significantly increased compared to the control group for comparable ranges of speed. This result is consistent with the findings of Nolan and Lees ${ }^{11}$ concerning the functional demand on the intact limb for transfemoral amputees.

The increase of mechanical work production by the intact limb partially compensates the decrease of mechanical work resulting from the affected limb. Donelan et al. ${ }^{21}$ already showed that the combined limbs method underestimates external mechanical work because during double support positive and negative work is performed simultaneously by the leading and the trailing legs.

One of the principal limitations of the present study is that we did not measure oxygen consumption during gait; it was therefore impossible to calculate the correlation between the increase of mechanical work and the increase of metabolic cost. However, as stated in the introduction, the measurement of gas exchange during gait necessitates a specific instrumentation and is often performed in treadmill. ${ }^{13,18}$ In contrast, the quantification of mechanical work can be made using the conventional gait analysis equipment and can therefore be integrated in the follow-up process of people with amputation.

Not surprisingly, average mechanical power during step-to-step transitions increased with walking velocity for both asymptomatic and amputee subjects. This confirms the results of previous studies on asymptomatic subjects $^{16,17}$ and amputees. ${ }^{18,22}$ But, as the present research is the first to apply the individual limb method to calculate mechanical work during step-to-step transition for people with transfemoral amputation, it provides an original insight into the asymmetric work produced by each limb for these patients. As already showed by Kuo et al., ${ }^{16}$ the total mechanical power is a good estimation of the resulting power from the three major joints of the lower limbs (ankle, knee and hip). Therefore, mechanical power analysis provides an overview of the combined actions of these joints in producing mechanical work useful to the locomotion. ${ }^{16}$

As regards the influence of walking velocity, mechanical work produced by the trailing limb during the double stance phase is clearly correlated with walking velocity. For asymptomatic people and for people with a unilateral transfemoral amputation, this work increased with walking velocity. However, the amount of mechanical work that can be produced during the propulsion by the affected limb is further limited at faster walking velocities. On the contrary, the mechanical work that must be produced by the intact limb in late stance rapidly increased with walking velocity showing the additional demand on the intact limb to ensure the progression of the body. This finding is consistent with the increase of metabolic cost observed for transfemoral amputees depending on walking velocity. ${ }^{2}$

As already stated in the literature, the double stance phase is a critical phase of gait for transfemoral amputees as it corresponds to the period when knee flexion must be initiated. ${ }^{2,10}$ Seroussi et al. ${ }^{8}$ observed an increase of concentric hip pull-off for the affected limb referring to the concentric work done by hip flexors during push off. Schmalz et al. ${ }^{2}$ also noted an increase of hip moment to initiate knee bending when shifting the prosthetic knee joint posteriorly. Considering the 
mechanical behaviour of a prosthetic knee, knee flexion initiation may require a flexor moment at the joint that can be obtained by generating a flexion moment at the hip. ${ }^{10}$ The results of the present study support the increase of hip moment at the prosthetic hip, which is also correlated with walking velocity. Hip moment values are in accordance with those of Sjödahl et al. ${ }^{23}$ even if they are difficult to compare due to different walking velocities. The hip moment generated at the residual limb reorients the ground reaction force in order to make it go behind the knee joint allowing knee bending. However, reorientation of the force has the direct consequence of decreasing mechanical work of the prosthetic leg, observed for the patients with transfemoral amputation, preventing them from producing as much work as asymptomatic subjects during push off. Thus, we can speculate that the reorientation of the ground reaction force can be achieved with strong muscle activity at the hip that can explain a part of the increased metabolic cost for transfemoral amputees as already hypothesized by Schmalz et al. ${ }^{2}$ and Seroussi at al. ${ }^{8}$

To conclude, the results confirm that the step-to-step transition is a critical phase of gait, and that measuring the mechanical work of the individual limbs can be a useful method that may correlate with the metabolic demand of walking in transfemoral amputees. In particular, the study shows the limitations undergone by transfemoral amputee, which results from a significant decrease of the mechanical work produced by the affected limb in opposition with an increase of this study at the intact limb during step-to-step transition compared to the control group. We also found that affected limbs were unable to increase the produced mechanical work with walking velocity in the same amount as the contralateral limb or the asymptomatic limbs. These limitations were also related to the increase of the hip flexion moment during the late stance phase, necessary to initiate prosthetic knee flexion. The process of prosthetic fitting and rehabilitation are dedicated to enhance gait to improve patients' autonomy in the society. To validate mechanical work of individual limbs as an indicator of the energetic efficiency of gait, an experiment combining metabolic demand quantification and mechanical work should be designed.

\section{Acknowledgements}

The authors thank Didier Azoulay and Christian Cazorla, prosthetists at CERAH/INI, and the patients who participated in the study.

\section{Declaration of conflicting interests}

The authors declare that there is no conflict of interest.

\section{Funding}

This study was financially supported by Proteor.

\section{References}

1. Waters RL and Mulroy S. The energy expenditure of normal and pathologic gait. Gait Posture 1999; 9: 207-231.

2. Schmalz T, Blumentritt S and Jarasch R. Energy expenditure and biomechanical characteristics of lower limb amputee gait: the influence of prosthetic alignment and different prosthetic components. Gait Posture 2002; 16: 255-263.

3. Gailey RS, Lawrence D, Burditt C, et al. The CATCAM socket and quadrilateral socket: a comparison of energy cost during ambulation. Prosthet Orthot Int 1993; 17: $95-100$

4. Graham LE, Datta D, Heller B, et al. A comparative study of oxygen consumption for conventional and energy-storing prosthetic feet in transfemoral amputees. Clin Rehabil 2008; 22: 896-901.

5. Boonstra AM, Schrama J, Fidler V, et al. Energy cost during ambulation in transfemoral amputees: a knee joint with a mechanical swing phase control vs a knee joint with a pneumatic swing phase control. Scand $J$ Rehabil Med 1995; 27: 77-81.

6. Traballesi M, Delussu AS, Averna T, et al. Energy cost of walking in transfemoral amputees: comparison between Marlo Anatomical Socket and Ischial Containment Socket. Gait Posture 2011; 34: 270-274.

7. Au S, Berniker M and Herr H. Powered ankle-foot prosthesis to assist level-ground and stair-descent gaits. Neural Netw 2008; 21: 654-666.

8. Seroussi RE, Gitter A, Czerniecki JM, et al. Mechanical work adaptations of above-knee amputee ambulation. Arch Phys Med Rehabil 1996; 77: 1209-1214.

9. Jaegers SMHJ, Arendzen JH and Jongh HJD. Prosthetic gait of unilateral transfemoral amputees: a kinematic study. Arch Phys Med Rehabil 1995; 76: 736-743.

10. Radcliffe CW. Four-bar linkage prosthetic knee mechanisms: kinematics, alignment and prescription criteria. Prosthet Orthot Int 1994; 18: 159-173.

11. Nolan L and Lees A. The functional demands on the intact limb during walking for active trans-femoral and trans-tibial amputees. Prosthet Orthot Int 2000; 24: $117-125$.

12. Gitter A, Czerniecki J and Weaver K. A reassessment of center-of-mass dynamics as a determinate of the metabolic inefficiency of above-knee amputee ambulation. $A m$ J Phys Med Rehabil 1995; 74: 332-338.

13. Detrembleur C, Vanmarsenille JM and Cuyper FD. Relationship between energy cost, gait speed, vertical displacement of centre of body mass and efficiency of pendulum-like mechanism in unilateral amputee gait. Gait Posture 2005; 21: 333-340.

14. McGeer T. Passive dynamic walking. Int J Rob Res 1990; 9: $62-82$.

15. Kuo AD. The six determinants of gait and the inverted pendulum analogy: a dynamic walking perspective. Hum Mov Sci 2007; 26: 617-656.

16. Kuo AD, Donelan JM and Ruina A. Energetic consequences of walking like an inverted pendulum: step-tostep transitions. Exerc Sport Sci Rev 2005; 33: 88-97.

17. Donelan JM, Kram R and Kuo AD. Mechanical work for step-to-step transitions is a major determinant of the metabolic cost of human walking. J Exp Biol 2002; 205: $3717-3727$. 
18. Houdijk H, Pollmann E, Groenewold M, et al. The energy cost for the step-to-step transition in amputee walking. Gait Posture 2009; 30: 35-40.

19. Pillet H, Bonnet X, Lavaste F, et al. Evaluation of force plate-less estimation of the trajectory of the centre of pressure during gait. Comparison of two anthropometric models. Gait Posture 2010; 31: 147-152.

20. Dempster W. Space requirements of the seated operator. Montgomery, OH: Wright-Patterson Air Force Base, 1955.

21. Donelan JM, Kram R and Kuo AD. Simultaneous positive and negative external mechanical work in human walking. J Biomech 2002; 35: 117-124.

22. Tesio L, Lanzi D and Detrembleur C. The 3-D motion of the centre of gravity of the human body during level walking. II. Lower limb amputees. Clin Biomech (Bristol, Avon) 1998; 13: 83-90.

23. Sjödahl C, Jarnlo GB, Söderberg B, et al. Kinematic and kinetic gait analysis in the sagittal plane of trans-femoral amputees before and after special gait re-education. Prosthet Orthot Int 2002; 26: 101-112.

\section{Appendix I}

\section{Details on the calculation of the mechanical power}

When walking, three forces are applied to the human body during the double support phase: $\vec{W}$ the gravity action modelled by a force applied at the centre of mass of the body; $\overrightarrow{F r}$ and $\overrightarrow{F l}$ the right and left ground reactions, respectively. Noting $\overrightarrow{\gamma_{G / R o}}$ the acceleration of the centre of mass and $m$ the total mass of the body, it is possible to express the dynamic equilibrium

$$
m \overrightarrow{\gamma_{G / R o}}=\vec{W}+\overrightarrow{F r}+\overrightarrow{F l}
$$

The energetic analysis of the system also allows writing

$$
P_{\text {kin }}=P_{\text {ext }}+P_{\text {int }}
$$

where $P_{\text {kin }}=d E k i n / d t$ is the kinetic power defined as the derivative of the kinetic energy, $P_{\text {ext }}$ is the power of external actions and $P_{\text {int }}$ is the power of internal actions.

Considering that the feet do not slide on the ground, the external power is reduced to the power of the gravity action, which is equal to $P(\vec{W})=\vec{W} \times \overrightarrow{V_{G / R o}}$ where $\overrightarrow{V_{G / R o}}$ is the velocity of the centre of mass of the body.

So, equation (2) becomes

$$
\frac{d E k i n}{d t}=\vec{W} \times \overrightarrow{V_{G / R o}}+P_{i n t}
$$

The total kinetic energy is composed of kinetic energy of the translation of the centre of mass and kinetic energy due to the rotations of the body segments. Making the hypothesis that the kinetic energy due to the rotations of the body segments can be neglected in front of the kinetic energy of the translation of the centre of mass, equation (2) can be written as follows

$$
\frac{d\left[\sum_{\text {segments }} \frac{1}{2} \times m_{i}\left(\overrightarrow{V_{G i / R o}}\right)^{2}\right]}{d t}=\vec{W} \times \overrightarrow{V_{G / R o}}+P_{\text {int }}
$$

Giving

$$
m \overrightarrow{\gamma_{G / R o}} \times \overrightarrow{V_{G / R o}}=\vec{W} \times \overrightarrow{V_{G / R o}}+P_{i n t}
$$

Combining equations (1) and (3), we obtain

$$
P_{\text {int }}=\overrightarrow{F r} \times \overrightarrow{V_{G / R o}}+\overrightarrow{F l} \times \overrightarrow{V_{G / R o}}
$$

So, the sum of the mechanical powers produced by each lower limb is an estimation of the mechanical power of the body.

The mechanical work of each individual limb was then assessed using the time integral of mechanical power profiles provided by each term of this sum. 